\title{
Solidarität statt Rassismus in Chemnitz
}

\section{Transformative Krisenbearbeitung in reflexiven Kollaborationen}

\author{
Stephan Liebscher ${ }^{1} \cdot$ Juliana Corvino $^{2} \cdot$ Lisa Hetmank $^{2}$ \\ Eingegangen: 7. April 2020 / Überarbeitet: 6. November 2020 / Angenommen: 7. November 2020 / Online publiziert: 17. November 2020 \\ (c) Der/die Autor(en) 2020
}

\section{Zusammenfassung}

Der Artikel lotet die Chancen und Limitationen von Kollaborationen zwischen lokalen zivilgesellschaftlichen Akteurinnen und Akteuren sowie Forschenden im sächsischen Chemnitz aus. Ein besonderes Augenmerk liegt auf der Analyse und Gestaltung solidarischer Formen des städtischen Zusammenlebens, die im Nachgang der rassistischen Ausschreitungen im Spätsommer 2018 sowie als Alternative zu „Neuen Rechten Bewegungen“ entwickelt wurden. In experimentellen Räumen und Praktiken können spontane solidarische Reaktionen auf offenen Rassismus auch jenseits von medialer Aufmerksamkeit aufgegriffen werden und über krisenhafte Momente hinaus potenziell Zusammenschlüsse von Akteurinnen und Akteure schaffen, die sich für ein solidarisches Miteinander einsetzen. Anhand eines Lehrforschungsprojektes wird das gemeinsame Arbeiten an Kritik der lokalen Krisenaufarbeitung, solidarischen Visionen und konkreten Handlungspfaden illustriert und reflektiert. Mit diesen Überlegungen aus der transformativen Forschung tragen wir zur Diskussion um die Verzahnung von angewandter und kritischer Geographie bei.

Schlüsselwörter \#wirsindmehr $\cdot$ Krise $\cdot$ Solidarische Lebensweise $\cdot$ Transformative Forschung $\cdot$ Postmigrantische Gesellschaft

\begin{abstract}
The article explores the opportunities and limitations of collaborations between local civil society actors and researchers in Chemnitz, Saxony. Particular attention is paid to the analysis and design of solidary forms of urban coexistence, which are being developed in the wake of the racism riots in late summer 2018 and as an alternative to New Right movements. In experimental spaces and practices, spontaneous solidary reactions to open racism can also be taken up beyond media attention and, beyond moments of crisis, potentially create associations of actors who are committed to a solidary coexistence. Using a teaching research project as example, the joint work on criticism of local crisis management, solidary visions, and concrete paths of action will be illustrated and deliberated. With these reflections from transformative research, we contribute to the discussion on the combination of applied and critical geography.
\end{abstract}

Keywords \#wirsindmehr - Crisis $\cdot$ Solidary mode of living $\cdot$ Transformative research $\cdot$ Postmigrant society

\section{Spätsommer 2018: Chemnitz in der Krise nach rassistischen Ausschreitungen}

Stephan Liebscher

s.liebscher@fu-berlin.de

1 Freie Universität Berlin, Malteserstraße

74-100, 12249 Berlin, Deutschland

2 TU Chemnitz, Chemnitz, Deutschland
Im Spätsommer 2018 erlangte Chemnitz nach rassistischen Ausschreitungen bundesweit und international öffentliche Aufmerksamkeit. Nachdem bei einer Auseinandersetzung auf dem Chemnitzer Stadtfest im August 2018 ein 35-Jähriger getötet und 2 asylsuchende Männer verhaftet worden waren, kam es an mehreren Tagen zu rassistischen Ausschreitungen und Hetzjagden auf (vermeintliche) Migran- 
tinnen und Migranten. Am 1. September riefen rechte Parteien und Gruppierungen wie die AfD, ProChemnitz und Pegida zu einem ,Trauermarsch“ auf, der von etwa 10.000 Menschen begleitet wurde (Friese et al. 2019, S. 7 f.). Die Ereignisse stürzten die ostsächsische Stadt in eine tiefe Krise (Intelmann 2019). Die Gegenreaktionen aus der Chemnitzer Zivilgesellschaft und von ortsansässigen Institutionen waren vielfältig und führten u. a. zu dem Konzert \#wirsindmehr mit namhaften Bands, an dem 65.000 Menschen aus ganz Deutschland teilnahmen und das tausendfach in sozialen Medien geteilt und diskutiert wurde.

Nachdem die öffentliche Aufmerksamkeit für die Geschehnisse in Chemnitz abgeebbt war, blieb es eine offene Frage, wie die spontanen Reaktionen in Alltagsleben und institutioneller Praxis in Chemnitz fortgeführt werden (können). Dies war der Ausgangspunkt des studentischen Forschungsprojektes mit dem Titel „Solidar-O-Mat“, das sich im Rahmen eines Seminars an der TU Chemnitz im Sommersemester 2019 mit kriseninduziertem solidarischen Handeln befasste. Angeregt durch den Forschungsansatz des Urban Transition Labs, das als Plattform für einen gemeinsamen Prozess der Erforschung und Konzipierung (Schäpke et al. 2017) solidarischen Handelns diente, sind 8 Studierende, 6 lokale Initiativen, 60 Demonstrierende und 1 Lehrender zusammengekommen. Die Autorinnen und der Autor des vorliegenden Artikels waren als Studierende bzw. als Lehrender in das Projekt, das im Folgenden vorgestellt wird, involviert.

Ausgehend von einer Kritik der konventionellen Versuche, mit der Krise in Chemnitz umzugehen (Abschn. 2), argumentieren wir für einen transformativen Modus der Krisenbearbeitung (Abschn. 3) und unterscheiden dabei 3 Ebenen der Kollaboration zwischen Zivilgesellschaft und Wissenschaft (Abschn. 4). Danach skizzieren wir den Forschungsprozess und diskutieren anhand der Projektergebnisse die Chancen und Limitationen der Kollaborationen (Abschn. 5 und 6).

\section{Kritik des konventionellen Modus der Krisenbearbeitung in Chemnitz}

In direkter Reaktion auf die rassistischen Ausschreitungen vom Spätsommer 2018 stellten sich Solidaritätsbekundungen sowie Fassungslosigkeit bei den Akteurinnen und Akteuren in Chemnitz ein. Etablierte ortsansässige Institutionen machten ihre Sichtweisen medial und im Stadtbild sichtbar. Mit den Hashtag-Kampagnen \#wirsindchemnitz oder \#wedergraunochbraun traten Bildungs- und Kulturinstitutionen sowie lokale Unternehmen gegen Rassismus und gegen Rechte ein. Auch aufseiten der Zivilgesellschaft und der lokalen Kulturszene artikulierte sich Empörung über den offenen Rassismus von rechten Gruppierungen und es erfolgte eine breite Mobilisierung für das Konzert \#wirsindmehr, das von lokalen Bands und Vereinen organisiert wurde. Als meistgenutzter Hashtag Deutschlands im Jahr 2018 (Spiegel Online 2018) entwickelte sich \#wirsindmehr zum Symbol für ,,postmigrantische Allianzen“ (Stjepandić und Karakayalı 2018).

Es steht außer Frage, dass die ereignisbezogene Empörung über rassistische Ausschreitungen zu begrüßen ist. Die spontane Empörung städtischer Institutionen und zivilgesellschaftlicher Gruppen sind jedoch als Ausdruck einer strukturkonservativen und stabilisierenden Krisenbearbeitung einzuordnen, die charakteristisch für kapitalistische Gesellschaften ist (Brand 2009, S. 3): Die Beschäftigung mit der Krise erfolgte bezogen auf Einzelereignisse und zeitlich begrenzt. Zudem wurde die Krise als externes Problem betrachtet, sodass eigene Verstrickungen in gesellschaftliche Machtverhältnisse unhinterfragt bleiben. So drängt sich gerade bei den bunten Fassadenbannern und Offenen Briefen, mit denen in Chemnitz auf die Ausschreitungen reagiert wurde, der Eindruck auf, es handele sich vor allem um Bemühungen, das Image und die Attraktivität der Stadt als Wirtschafts- und internationaler Universitätsstandort zu retten. Rassismus wurde einmal mehr als Problem rechter Gruppierungen und Netzwerke und die Ausschreitungen als Einzelereignis, nicht als gesellschaftliches Verhältnis konstruiert, das bis in die eigenen Strukturen hineinwirkt (Friese et al. 2019, S. 8). Folglich blieben Problemanalysen und Praktiken, die über den Krisenmoment hinausweisen, Randnotizen.

\section{Für einen transformativen Modus der Krisenbearbeitung}

Für die Bearbeitung der Chemnitzer Krise schlagen wir einen transformativen Modus vor, der sich anhand von 2 Kernmerkmalen charakterisieren lässt:

- Kollaborativ-reflexiv: Transformativ Forschende intendieren, einen aktiven Beitrag zu Transformationsprozessen zu leisten (Räuchle und Schmiz 2019). Um die unterschiedlichen Verwicklungen in Machtverhältnisse zu verdeutlichen, scheint der Ansatzpunkt von Schäpke et al. (2017, S. 35) gewinnbringend, zivilgesellschaftliche Akteurinnen und Akteuren als radikale Vorreiterinnen und Vorreiter von Transformation und Institutionen als Innovatorinnen des Mainstreams zu betrachten. Dies impliziert, Forschung als Schaffensprozess zu verstehen, in welchem die Kritikerin bzw. der Kritiker die Position des Begutachtens zeitweise verlässt und übergeht $\mathrm{zu}$ einem Tun, das ,einen pragmatischen Rahmen für Veränderung“" (Terkessidis 2015, S. 12) schafft. Statt sich in distanzierter Haltung zur empirischen Gemengelage zu 
äußern, steht die Verwicklung von Forschenden in lokale Anwendungsfelder im Blick.

- Solidarisch: Solidarische Formen der Krisenbearbeitung sind auf Veränderungen in Richtung einer sozialökologischen Transformation ausgerichtet. Mit dem Begriff der "solidarischen Lebensweise“ umschreibt das I.L.A. Kollektiv (2019) eine mögliche inhaltlich-normative Ausrichtung von Transformationen, die folgenden Prinzipien folgt:

„Ein Leben, das nicht auf Kosten anderer Menschen, der Natur oder zukünftiger Generationen geht. Das ist die Voraussetzung für solidarische Lebensweisen - die rote Linie, hinter die reale Utopien nicht zurückfallen wollen“ (I.L.A. Kollektiv 2019, S. 18).

Dieser Rahmen bietet Orientierung für Veränderungen, die sowohl Strukturen und Institutionen als auch das Alltagsleben umfassen. Krisenphasen können als Auslöser betrachtet werden, um gesellschaftspolitische wie wissenschaftliche Anstrengungen auf die Analyse und Umgestaltung von Gesellschaftsverhältnissen und Alltagspraktiken zu richten (Brand 2009, S. 11). Voraussetzung für derartige Praktiken im Sinne der solidarischen Lebensweise ist eine Krisendeutung, welche rassistische Ausschreitungen und damit zusammenhängende Krisenphasen in den Kontext grundlegender Krise kapitalistischer Gesellschaftsverhältnisse einordnet.

In diesem Sinne intervenieren Forschende und zivilgesellschaftliche Akteurinnen und Akteure in gesellschaftliche Transformationsprozesse, wobei ihnen die solidarische Lebensweise als Orientierungsrahmen für Alltagshandeln dient. Dabei ergibt sich der gesellschaftskritische Blick aus dieser normativen Zielsetzung, während der Anwendungsbezug in Kollaborationen zwischen Wissenschaft und Zivilgesellschaft definiert werden kann.

\section{Ebenen der Kollaboration zwischen Zivilgesellschaft und Wissenschaft}

Das gemeinsame Verständnis von komplexen, sogenannten „multiplen“ Krisendeutungen (Brand 2009) kann eine diskursive Basis für Kollaborationen zwischen Akteurinnen und Akteuren aus Zivilgesellschaft und Wissenschaft sein. Es weitet den Blick über ein Krisenphänomen hinaus auf deren Wechselwirkungen mit anderen Krisendimensionen kapitalistischer Gesellschaften. Dabei werden die wiederkehrenden Krisenmomente als „Ausdruck von tief in den Gesellschaften verankerten Produktions- und Lebensweisen, die sozial spaltend und Natur zerstörend sind“ (ebd., S. 5), betrachtet. Demnach lässt sich die Krise von Chemnitz nicht als isoliertes Phänomen betrachten, sondern nur in Zusammenhang mit weiteren ökonomischen, sozialen und institutionellen Aspekten. Eine derartige Krisendeutung ist die Voraussetzung für transformative Formen solidarischer Krisenbearbeitung.

Inhalte der Kollaboration können sich rund um die Frage „Wie lässt sich die Stadt Chemnitz solidarisch gestalten?“ ergeben. In der transformativen Forschung wird zur Bearbeitung die Aufteilung in 3 Wissensarten vorgeschlagen: Kritik, Utopie und Transformation (Schäpke et al. 2017, S. 9). In einem aufeinander aufbauenden Lern- und Arbeitsprozess beschäftigen sich die Akteurinnen und Akteure konkret mit den Fragen „Was läuft schief? Wie soll die Zukunft (nicht) sein? Wie lässt sich die Zukunft gestalten und wo fangen wir an?" Dabei wird deutlich, dass Kritik immer nur der 1. Schritt sein kann, dem das utopische Denken sowie das Planen konkreter Veränderungsschritte und Ausprobieren verschiedener Handlungspfade folgen müssen.

Das organisatorische Setting der Kollaboration zwischen Forschenden und Zivilgesellschaft wird mit dem Forschungsansatz des Urban Transition Labs gerahmt. Schäpke et al. fassen diese ,als hybride, flexible und transdisziplinäre Plattformen [auf], [die] Zeit und Raum zum Lernen, zur Reflektion und zur Entwicklung von alternativen Lösungen für [P]robleme zur Verfügung stellen" (2017, S. 35). Entlehnt aus dem privatwirtschaftlichen Bereich können Labor-Ansätze auch für stadtpolitische und sozialgeographische Zwecke eingesetzt werden, indem der Fokus auf soziale und politische Prozesse gelegt wird (Räuchle und Schmiz 2019). Die Labs haben den Anspruch, sowohl forschungsrelevantes als auch handlungspraktisches Wissen zu Transformationsprozessen zu generieren.

\section{Transformativer Forschungsprozess}

Die studentische Forschungsgruppe widmete sich im Projekt „Solidar-O-Mat“ zivilgesellschaftlichen Perspektiven auf und Handlungen nach der Chemnitzer Krise. Angeregt durch den Forschungsansatz des Urban Transition Lab unterteilte sich der transformative Forschungsprozess in 3 Phasen: Zunächst erhoben sie empirische Daten zur Chemnitzer Zivilgesellschaft. Bei einer Demonstration am 1. Mai 2019 wurden Postkarten verteilt und die Demonstrierenden aufgefordert, die Aussage ,Solidarität in Chemnitz - Frag' was oder sag' was!" schriftlich zu kommentieren. Neben den knapp 60 erhobenen Statements wählten die Forschenden in einem Expertininterview mit einer Vertreterin einer großen Chemnitzer Organisation 5 zivilgesellschaftliche Vorreiterinnen für Solidarität aus: Agiua e. V., Bandbüro Chemnitz e. V., Buntmacher/innen, different people e.V. und Spinnerei e. V. Diese wurden nach Solidaritätsdefinitionen, Arbeitsfeldern, Grundprinzipien und Zukunftsvisionen 
schriftlich befragt (Diekmann 2008, S. 514ff.). Die Daten sind inhaltsanalytisch (Mayring 1994) ausgewertet worden.

Die 2. Phase stand im Zeichen der Kollaboration von Forschenden und den befragten Initiativen. Das Ziel der Zusammenarbeit bestand in der Erarbeitung transformativer Handlungspfade und konkreter prototypischer Outputs, welche potenziell dazu beitragen sollen, die Solidarität in Chemnitz zu erhöhen. Auf Basis der empirischen Daten konzipierten Forschende und die Initiativen eine Informationsbroschüre zu Engagementmöglichkeiten sowie eine kritisch kartographische (Glasze 2009) Repräsentation des Chemnitzer Stadtbildes. Die Forschungsgruppe erstellte daraufhin das Design und die Inhalte der Broschüre.

Der transformative Charakter des Forschungsprozesses ist, 3., anhand ethnografischer (Selbst-)Beobachtungen (Breidenstein et al. 2013) der Forschungsgruppe sowie des Lehrenden reflektiert worden.

\section{Das Projekt „Solidar-O-Mat": Solidarisches Zusammenleben in Chemnitz analysieren und gestalten}

Im Folgenden stellen wir anhand des Forschungsprojektes ,Solidar-O-Mat“ mögliche Handlungspfade und konkrete prototypische Outputs eines transformativen Modus der Krisenbearbeitung dar. Zudem reflektieren wir die Chancen und Limitationen der Kollaboration zwischen Zivilgesellschaft und Wissenschaft.

\section{Diskursive Basis: Alternative Krisendeutung und Alltagssolidarität}

Die empirische Datengrundlage für Kollaborationen zwischen Forschenden und lokalen Initiativen bildete die Analyse der Verständnisse von Solidarität und der Krisenereignisse im Spätsommer 2018 aufseiten der zivilgesellschaftlichen Akteurinnen und Akteure. Die erhobenen Daten zeugen, 1., von der Existenz alternativer Krisendeutung in der Chemnitzer Zivilgesellschaft. So finden sich Problembeschreibungen rassistischer Gesellschaftsverhältnisse: Gerade migrantisch positionierte Vereine verweisen auf langjährige Kontinuitäten von Rassismus in Chemnitz und Sachsen und die damit verbundenen Unsicherheiten im Alltagsleben der Betroffenen (Vu und Grunert 2018). Eines der Statements verweist darauf, dass im Chemnitzer Alltag Menschen, die als Migrantinnen oder Migranten aufgefasst werden, generell ausgeschlossen werden:

Missstände in Chemnitz: kaum Einbindung von Personen mit Migrationshintergrund in Bürgergespräche und rechte Sprache wird salonfähig.
Ein anderes Statements nimmt, 2., eine Entsolidarisierung wahr und verortet diese im Zusammenhang mit neoliberalen Ordnungen:

Solidarität ist die Grundhaltung, die in Chemnitz fehlt und die heute im Zuge des Neoliberalismus aufgelöst wird. Solidarität ist mehr als gemeinsam auf einem Markt stehen, Solidarität bedenkt täglichen Kampf.

Das Statement übt damit Kritik an einem ereignisbezogenen Solidaritätsbegriff und stellt diesem einem alltagspraktischen gegenüber. Dies geht bei den Befragten einher mit einer Hinwendung zur Stadt Chemnitz allgemein: Wurden anfänglich Abgrenzungen gegen rechte Gruppierungen und gegen Rassismus zu einer einenden Basis für Kooperationen vor Ort, geriet im Fortgang zunehmend das Engagement für das städtische Zusammenleben im Allgemeinen in den Vordergrund. Dies bestätigt sich an folgender Einschätzung eines befragten Vereins und eines Demo-Statements:

Die Ereignisse im August sind eher ein Katalysator anstatt Bremse, da die Stadt im Mittelpunkt steht.

Solidarität herrscht unter denjenigen, die bereits aktiv sind. Für den Großteil der Menschen fehlt jedoch der Bezug zu den anderen, das Gefühl für das gemeinsame Menschsein, das für Solidarität benötigt wird.

Auch der erhöhte Zulauf zu den befragten Initiativen aus dem Kulturbereich, die sich in ihren Aktivitäten nicht explizit mit antirassistischen Themen beschäftigen, weist in diese Richtung. Insgesamt zeigt sich anhand der empirischen Daten, dass sowohl Krisendeutungen, die auf den Zusammenhang zwischen rassistischen Einzelereignissen und Entsolidarisierungen verweisen, als auch alltagsbezogene Solidaritätsverständnisse in der Chemnitzer Zivilgesellschaft vorhanden sind. Auch wenn die erhobenen Daten nur einen kleinen Teil der Stadtgesellschaft abdecken, stellen sie eine diskursive Basis dar, auf welcher Kollaborationen zwischen Forschenden und zivilgesellschaftlichen Vorreiterinnen und Vorreitern aufbauen können.

\section{Inhalte: Eine Broschüre für sichtbare Solidarität und mehr Engagement}

Wurde bereits in den Postkarten-Statements den lokalen Initiativen eine relevante Rolle für ein solidarisches Miteinander zugeschrieben, konkretisierten sich in der anschlieBenden Befragung der Initiativen mögliche Wege zur Etablierung transformativer Praktiken und der Stärkung von Alltagssolidarität:

Wir haben die Hoffnung, dass das Engagement verschiedenster Initiativen langfristig eine lebendige demokratische Alltagskultur in unserer Stadt aufbaut, die zur Stabilität der Gesellschaft beiträgt und die zuletzt entstandenen Gräben wieder schließt. 
Ausgehend von diesen Befunden definierte die Forschungsgruppe 2 Handlungspfade (Wittmayer et al. 2011) zur Stärkung eines solidarischen Miteinanders, für die sie auch vonseiten der Vereine und Initiativen Zuspruch erhielt: die Sichtbarkeit solidarisch ausgerichteter Initiativen in Chemnitz erhöhen und für das Engagement in diesen mobilisieren. Das Planen und das Ausprobieren dieser Handlungspfade gestalteten sich als kreativer Suchprozess. Zunächst entstand innerhalb der Projektgruppe die Idee, durch eine Art Übersichts- und Kontaktplattform einen transformativen Beitrag zu leisten. Daraus ergab sich der Projekttitel „Solidar-O-Mat“, welches nach dem Vorbild des Wahl-O-Mats der Bundeszentrale für politische Bildung (www.wahl-o-mat.de/) gestaltet werden und Interessierten in Übereinstimmung mit ihren Vorlieben passende Chemnitzer Initiativen vorschlagen sollte. In Diskussionen mit den Befragten und weiteren Initiativen bei einer lokalen Demokratiekonferenz sowie über einen Mailverteiler eines großen Netzwerkes stieß diese Idee auf breite Zustimmung und großes Interesse. Als 1. Prototypen erstellten die Forschenden anhand der in den Befragungen lokaler Initiativen und Vereine gewonnenen Daten eine 16-seitige Broschüre.

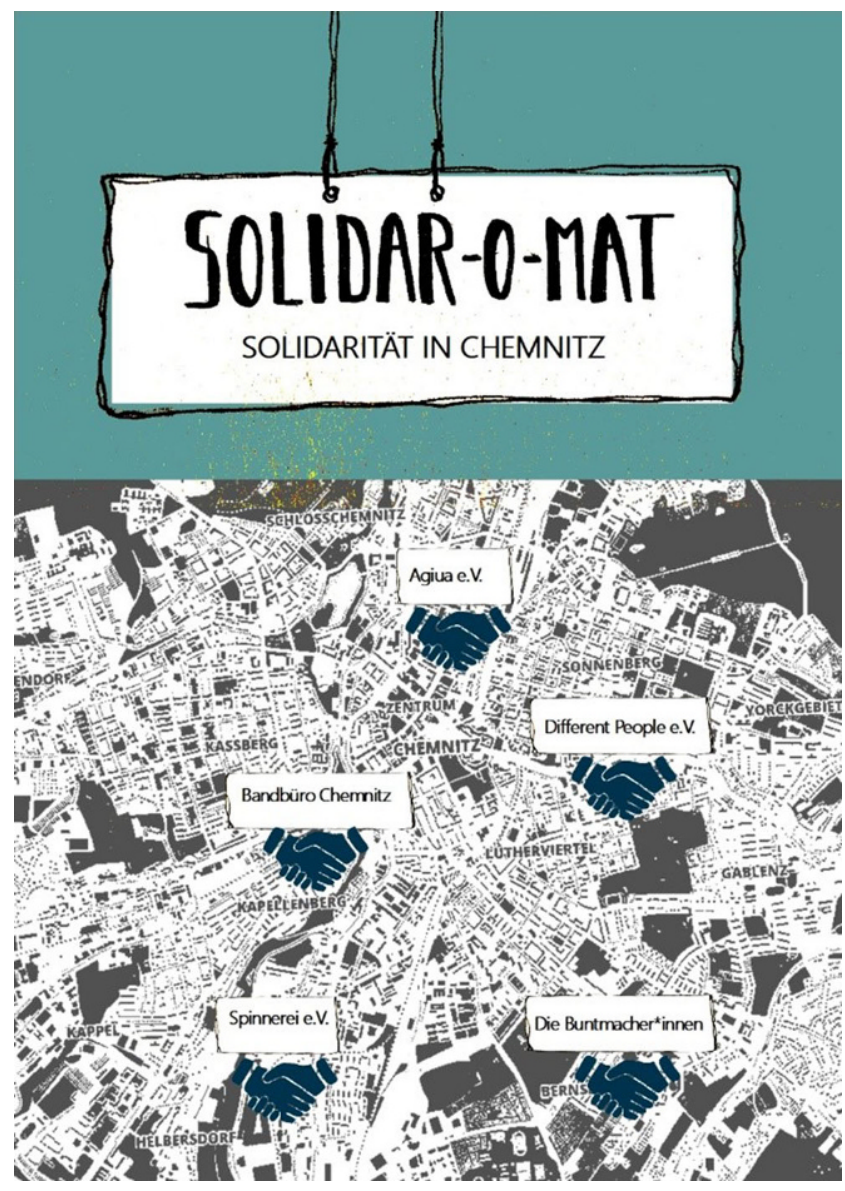

Abb. 1 Titelbild „Solidar-O-Mat“. (Quelle: Eigene Darstellung, basierend auf Daten von OpenStreetMap.org-Mitwirkende, CC-BY-SA 2.0)
Der 1. Handlungspfad schlägt sich vor allem im Titelbild der Broschüre nieder (vgl. Abb. 1). Durch die Kombination aus kartographischer und thematischer Verortung der Initiativen, die sich in Chemnitz für Solidarität einsetzen, wird eine strategische Festschreibung vorgenommen (Casas-Cortés et al. 2014, S. 303). Der 2. Handlungspfad wird durch Informationsseiten zu den 5 Initiativen, die das Kernstück der Broschüre darstellen, bedient (vgl. beispielhaft Abb. 2). Sie beinhalten Textelemente zu Zielen und Aktivitäten, Engagement- und Kontaktmöglichkeiten sowie Visionen und Solidaritätsverständnissen. Anhand der Übersichten können Interessierte durch eine Auswahl Chemnitzer Initiativen navigieren und die für sie passende Gruppe identifizieren. Im Sinne des Konzeptes der ,solidarischen Lebensweise“ bietet die Broschüre Nutzenden Orientierung im Nachgang der Chemnitzer Krise und zeigt auf, dass sich lokale Initiativen für ein solidarisches Miteinander einsetzen.

Die Broschüre stieß bei Chemnitzer Initiativen auf großes Interesse. Wie die Chemnitzer Stadtgesellschaft die Broschüre annahm, entzieht sich der Kenntnis der Autorinnen und des Autors.

\section{Reflektion des Settings: Urban Transition Lab als geeignete Plattform für transdisziplinäre Kollaboration?}

Im Verlauf des kollaborativen Forschungs- und Gestaltungsprozesses, der durch den Forschungsansatz Urban Transition Lab gerahmt war, wurden sowohl das transformative Potenzial deutlich, das sich aus gemeinsamen Problemdeutungen und Definitionen von Handlungspfaden ergibt, als auch Herausforderungen, die aus bestehenden institutionellen Mustern einer Lehrforschungsveranstaltung erwachsen.

Die Postkartenaktion sowie die Initiativenbefragung ermöglichten eine gemeinsame Deutung der Chemnitzer Krise. Damit haben die Forschenden ihre Definitionsmacht hinsichtlich relevanter Probleme und möglicher Lösungen abgegeben und gewissermaßen den Prozess der Wissensproduktion ,demonopolisier[t]“ (Bayer und Terkessidis 2018, S. 203). Auf beiden Seiten löste dies Lernprozesse in Bezug auf die institutionelle Forschungspraxis aus: Während die Forschenden mit der transformativen Methodologie die Erfahrung machten, dass auch aus der Universität - mit Schäpke gesprochen: den Institutionen des Mainstreams - heraus Möglichkeiten der Mitgestaltung des Stadtlebens vorhanden sind, erfuhren die Vereine - als radikale Vorreiterinnen - die Universität als Kooperationspartnerin in für sie relevanten Anliegen.

Die Anwendung der transformativen Methodologie war allerdings auch durch „dominante Orientierungen“ (Brand 2009, S. 13) der Institution Universität geprägt, die ihrerseits die Kollaboration mit der Zivilgesellschaft auf spezifische Weise gestalteten und begrenzten. Die Initiative zur 


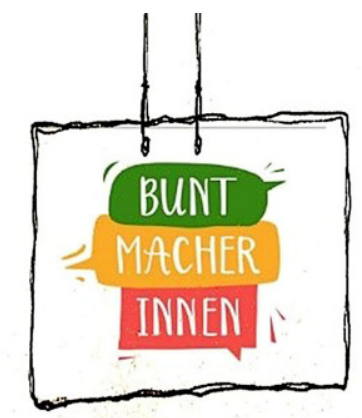

AUSTAUSCH AUF AUGENHÖHE

„Die positiven Wirkungen gelebter Solidarität stärken nachhaltig die Strukturen unseres demokratischen Miteinanders"

WIR

ÜBER

UNS

Wir sind eine bunte Mischung Chemnitzer Bürger*innen die sich gegründet haben, um das Bewusstsein für die Bedeutung demokratischer Werte in der Stadtgesellschaft zu stärken und die aufgebrochenen Gräben durch Dialog auf Augenhöhe zu schließen.

Wir möchten den offenen und zugewandten Austausch zwischen den Generationen über Vergangenheit, Gegenwart und Zukunft unseres Miteinanders befördern. Von entscheidender Bedeutung ist uns dabei die Belebung und Stärkung der demokratischen Alltagskultur und des öffentlichen Debattenraumes. Die Ausrichtung unserer Arbeit ist grundlegend positiv: wir möchten ein gemeinschaftsstiftendes Bekenntnis FÜR etwas.

WAS WIR MACHEN

Wahlkampf für die Demokratie"_Wir möchten die Wahlbeteiligung erhöhen und den Bürger*innen die Wichtigkeit der Beteiligung aufzeigen. Dazu gehören Dialog-Bazare, Parteineutraler Haustürwahlkampf, Festival zur Mobilisierung von Erst- und Jungwähler*innen sowie Filmpräsentation + Diskussion. .Lichterwege Chemnitz"_9. November Aufruf zur Stärkung der Werte unseres Zusammenlebens. Bekenntnis zu Toleranz, Respekt, Solidarität, Weltoffenheit und Vielfalt. Wege zu Erinnerungsorten untermalt von Musik und Sprechbeiträgen.
Bei uns ist es möglich, sich je nach Interessen, Möglichkeiten und Talenten, organisatorisch, kreativ usw. sowohl kurzfristig als auch langfristig einzubringen. Wir freuen uns ausdrücklich über Ideen, frischen Input und Energie, jegliche tatkräftige Unterstützung (ob personell oder materiell) bei unseren Projekten. Also, einfach mal melden. Wir haben immer ein offenes Ohr.

UNSERE Durch Zusammenarbeit mit Initiativen und Vereine möchten wir eine VISION vielstimmige, breit aufgestellte und beständige zivilgesellschaftliche und demokratische Streit- und Gesprächskultur fördern. Dies ist zur Gestaltung einer lebenswerten Zukunft in unserer Stadt unerlässlich
Das Unterstützen von Schwachen und Bedrängten, ein freiwilliges Eintreten für Minderheiten und Randgruppen verstehen wir als allgemeine gesellschaftliche Pflicht, zu der auch Zivilcourage zählt. Solidarität bildet für uns einen zentralen Begriff, solidarische Gesten können, vor allem im Kleinen, im Alltäglichen Gemeinschaft stiften, ein Vertrauen herstellen und im Sozialen und Gesellschaftlichen verbindend wirken.

\section{WOLLEN}

Abb. 2 Die Buntmacher/innen im „Solidar-O-Mat“. (Eigene Darstellung; Bildquelle: facebook.com/Buntmacherinnen/)

Durchführung eines Urban Transition Lab ging vor allem von den Forschenden aus, die einen Rahmen für einen transformativen Forschungsprozess schaffen wollten. Dadurch waren jedoch die Aufgaben und das Verantwortungsbewusstsein für den Prozess unterschiedlich verteilt. Aufseiten des Lehrenden entstand das konkrete Interesse an solidarischer Forschung und Mitgestaltung in Chemnitz hauptsächlich in Verbindung mit seiner Lehrverpflichtung. Entlang der Semesterzeiten sind damit Zeitpunkt, Start und Ende des Labs festgelegt worden. Die studentische Forschungsgruppe wiederum entschied sich angesichts der Semesterdauer für eine zielgerichtete Zusammenarbeit mit der Zivilgesellschaft und stellte das Lab-Format als solches sowie die Rollenverteilung zwischen Forschenden und Zivilgesellschaft nicht weiter zur Disposition. In diesem Spannungsfeld zwischen Bedarfen der Initiativen und institutionellen Mustern der Lehrforschung stellten wir uns fortwährend die Frage, inwiefern wir bereit sind, unseren Alltag für das $L a b$ zu ändern. Letztlich erkannten wir, dass diese Fra- ge auch ein Bestandteil der Aushandlungen mit den lokalen Akteurinnen und Akteuren sein kann.

\section{Fazit}

Anhand des Projektes „Solidar-O-Mat“ wurde gezeigt, wie im Zusammenwirken von Forschenden, Lehrendem und städtischer Zivilgesellschaft alltagspraktische Strategien zur Sichtbarmachung von solidarisch-ausgerichteten Initiativen entwickelt und erprobt wurden. Die Chemnitzer Krise nahmen die Autorinnen und der Autor dabei als Möglichkeit, dominante Orientierungen in einer Lehr- und Forschungsinstitution $\mathrm{zu}$ hinterfragen. (Rassismus-)Kritische Analysen des Umgangs etablierter Institutionen mit rassistischen Gesellschaftsverhältnissen stellten eine wichtige, aber nicht die einzige Säule unserer Wissensproduktion dar. Das transformative Vorgehen bot uns die Möglichkeit, von der Zivilgesellschaft ausgehende Problemdeutungen 
und Handlungspfade in akademischer Forschung und Lehre aufzugreifen. Die spezifischen Begrenzungen, die in der Umsetzung des Labs sichtbar wurden, offenbaren weiteren Forschungs- und Gestaltungsbedarf zu Formaten transdisziplinärer Kollaborationen.

Danksagung Wir möchten uns gerne bei den Befragten an der Demonstration am 1. Mai 2019 sowie den lokalen Initiativen in Chemnitz bedanken, die durch ihre Kollaboration die Erstellung des ,,Solidar-OMaten" erst möglich gemacht haben. Außerdem gilt dem Netzwerk für Kultur- und Jugendarbeit unser Dank, durch deren Vernetzungsangebot der Kontakt zu den Initiativen aufgebaut werden konnte. Herzlichen Dank auch an die wertvollen Kommentare der anonymen Reviewerinnen und Reviewer.

Funding Open Access funding enabled and organized by Projekt DEAL.

Open Access Dieser Artikel wird unter der Creative Commons Namensnennung 4.0 International Lizenz veröffentlicht, welche die Nutzung, Vervielfältigung, Bearbeitung, Verbreitung und Wiedergabe in jeglichem Medium und Format erlaubt, sofern Sie den/die ursprünglichen Autor(en) und die Quelle ordnungsgemäß nennen, einen Link zur Creative Commons Lizenz beifügen und angeben, ob Änderungen vorgenommen wurden.

Die in diesem Artikel enthaltenen Bilder und sonstiges Drittmaterial unterliegen ebenfalls der genannten Creative Commons Lizenz, sofern sich aus der Abbildungslegende nichts anderes ergibt. Sofern das betreffende Material nicht unter der genannten Creative Commons Lizenz steht und die betreffende Handlung nicht nach gesetzlichen Vorschriften erlaubt ist, ist für die oben aufgeführten Weiterverwendungen des Materials die Einwilligung des jeweiligen Rechteinhabers einzuholen.

Weitere Details zur Lizenz entnehmen Sie bitte der Lizenzinformation auf http://creativecommons.org/licenses/by/4.0/deed.de.

\section{Literatur}

Bayer N, Terkessidis M (2018) Antirassistisches Kuratieren im Museum der Vielheit. In: Foroutan N, Karakayalı J, Spielhaus R (Hrsg) Postmigrantische Perspektiven: Ordnungssysteme, Repräsentationen, Kritik. Campus, Frankfurt, S 191-206

Brand U (2009) Die Multiple Krise. Dynamik und Zusammenhang der Krisendimensionen, Anforderungen an politische Institutionen und Chancen progressiver Politik. Heinrich-Böll-Stiftung. https:// www.boell.de/sites/default/files/multiple_krisen_u_brand_1.pdf. Zugegriffen: 1. Apr. 2020

Breidenstein G, Hirschauer S, Kalthoff H, Nieswand B (2013) Ethnografie: Die Praxis der Feldforschung. UTB, Stuttgart

Casas-Cortés M, Cobarrubias S, Heller C, Pezzani L (2014) Kollidierende Kartografien, migrating maps. In: Heimeshoff L-M, Hess S, Kron S, Schwenken H, Trzeciak M (Hrsg) Grenzregime II: Migration - Kontrolle - Wissen. Transnationale Perspektiven. Assoziation A, Berlin, S 301-324

Diekmann A (2008) Empirische Sozialforschung: Grundlagen, Methoden, Anwendungen, 2. Aufl. Rowohlt, Reinbek bei Hamburg
Friese H, Nolden M, Schreiter M (2019) Chemnitz im Herbst. In: Friese H, Nolden M, Schreiter M (Hrsg) Rassismus im Alltag: Theoretische und empirische Perspektiven nach Chemnitz. transcript, Bielefeld, S 7-13

Glasze G (2009) Kritische Kartographie. Geogr Z 97:181-191

Intelmann D (2019) Sieben Thesen zur urbanen Krise von Chemnitz. Sublurban Z Krit Stadtforsch 7:189-202. https://doi.org/10. 36900/suburban.v7i1/2.468

Kollektiv ILA (2019) Das gute Leben für alle: Wege in die solidarische Lebensweise. Oekom, München

Mayring P (1994) Qualitative Inhaltsanalyse. In: Boehm A, Mengel A, Muhr T (Hrsg) Texte verstehen: Konzepte, Methoden, Werkzeuge. Univ.-Verl., Konstanz, S 159-175

Räuchle C, Schmiz A (2019) Reallabore in Ankunftsquartieren. IMIS Working Paper 03. Institut für Migrationsforschung und Interkulturelle Studien (IMIS) der Universität Osnabrück. https://www. imis.uni-osnabrueck.de/fileadmin/4_Publikationen/PDFs/IMIS_ WP03_Reallabore_RaeuchleSchmiz.pdf. Zugegriffen: 1. Apr. 2020

Schäpke N, Stelzer F, Bergmann M et al (2017) Reallabore im Kontext transformativer Forschung: Ansatzpunkte zur Konzeption und Einbettung in den internationalen Forschungsstand. IETSR Discussion Papers. Institute for Ethics and Transdisciplinary Sustainability Research, Leuphana University, Lüneburg https://doi. org/10.13140/rg.2.2.28604.23687

Spiegel Online (2018) WirSindMehr war der populärste DebattenHashtag. https://www.spiegel.de/consent-a-?targetUrl=https\%3A $\% 2 \mathrm{~F} \% 2$ Fwww.spiegel.de $\% 2$ Fnetzwelt $\% 2$ Fweb $\% 2$ Ftwitter-dassind-die-erfolgreichsten-debatten-hashtags-2018-a-1242047. html. Zugegriffen: 4. Juli 2020

Stjepandić K, Karakayalı S (2018) Solidarität in postmigrantischen Allianzen: Die Suche nach dem Common Ground jenseits individueller Erfahrungskontexte. In: Foroutan N, Karakayali J, Spielhaus R (Hrsg) Postmigrantische Perspektiven: Ordnungssysteme, Repräsentationen, Kritik. Campus, Frankfurt, S 237-252

Terkessidis M (2015) Kollaboration. Suhrkamp, Berlin

Vu V, Grunert J (2018) Chemnitz: Auf sich allein gestellt. https:// www.zeit.de/gesellschaft/zeitgeschehen/2018-08/chemnitzausschreitungen-rassismus-betroffene-erfahrungen. Zugegriffen: 4. Juli 2020

Wittmayer J, van Steenbergen F, Quist J et al (2011) The Community Arena: A co-creation tool for sustainable behaviour by local communities. Methodological guidelines. Deliverable 4.1-Guidelines for the implementation of pilot projects. https:// www.incontext-fp7.eu/sites/default/files/D4-1_Methodological \%20guidelines_final.pdf. Zugegriffen: 1. Apr. 2020

Stephan Liebscher Wissenschaftlicher Mitarbeiter AG Globalisierung, Transformation, Gender, Institut für Geographische Wissenschaften

Juliana Corvino Masterstudierende der Interkulturellen Kommunikation mit Schwerpunkt auf Lateinamerika

Lisa Hetmank Studentin der Europastudien mit kulturwissenschaftlicher Ausrichtung 